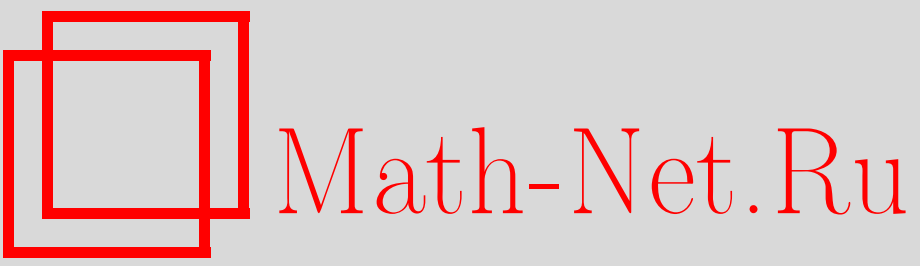

А. П. Янковский, О некоторых свойствах решения задачи равнонапряженного армирования изгибаемых металлокомпозитных пластин, работающих в условиях установившейся ползучести, Вестн. Сам. гос. техн. ун-та. Сер. Физ.-мат. науки, 2011, выпуск 2(), 62-73

DOI: https://doi.org/10.14498/vsgtu922

Использование Общероссийского математического портала Math-Net.Ru подразумевает, что вы прочитали и согласны с пользовательским соглашением http: //www. mathnet.ru/rus/agreement

Параметры загрузки:

IP: 34.229 .45 .116

26 апреля 2023 г., 10:49:03 
УДК 539.376

\title{
О НЕКОТОРЫХ СВОЙСТВАХ РЕШЕНИЯ ЗАДАЧИ РАВНОНАПРЯЖЁННОГО АРМИРОВАНИЯ ИЗГИБАЕМЫХ МЕТАЛЛОКОМПОЗИТНЫХ ПЛАСТИН, РАБОТАЮЩИХ В УСЛОВИЯХ УСТАНОВИВШЕЙСЯ ПОЛЗУЧЕСТИ
}

\section{А.П. Янковский}

Институт теоретической и прикладной механики им. С. А. Христиановича СО РАН, 630090, Новосибирск, ул. Институтская, 4/1.

E-mail: nemirov@itam.nsc.ru

\begin{abstract}
Сформулирована задача равнонапряжённого армирования (РА) поперечно изгибаемых пластин произвольной формы, работающих в условиях установившейся ползучести материалов всех компонент композиции. Проведён качественный анализ системы разрешающих уравнений. Исследованы свойства решений рассматриваемой задачи $\mathrm{PA,} \mathrm{в} \mathrm{частности,} \mathrm{определены} \mathrm{граничные} \mathrm{условия,} \mathrm{при} \mathrm{ко-}$ торых гладкие решения такой задачи не существуют, и изучены особенности поведения решения в окрестности жестко защемленных кромок пластины. Показаны возможсности существования нескольких альтернативных решений задачи РА при одних и тех же входных данных и управления полученными РАпроектами.
\end{abstract}

Ключевые слова: металлокомпозиты, равнонапряжённое армирование, изгибаемые пластины, установившаяся ползучесть, свойства решения, качественный анализ.

Введение. Одним из наиболее естественных прочностных критериев рационального проектирования тонкостенных элементов конструкций является требование равнонапряжённости силовых элементов - арматуры (волокон, проволок) - вдоль их траекторий, так как при этом несущая способность арматуры используется наиболее полно. При длительной эксплуатации изделия в случае стационарного термосилового нагружения подавляющую часть времени металлокомпозитная конструкция работает в условиях установившейся ползучести [1], поэтому актуальной является проблема равнонапряжённого армирования (PA) тонкостенных конструкций, работающих в условиях установившейся ползучести материалов всех компонент композиции.

В связи с этим настоящая работа посвящена исследованию задачи РА поперечно нагруженных изгибаемых металлокомпозитных пластин, работающих в условиях установившейся ползучести всех фаз композиции, и в этом смысле продолжает исследования, опубликованные в [2], где рассматривался частный случай задачи РА об осесимметричном изгибе кольцевых металлокомпозитных пластин.

1. Система исходных уравнений и граничные условия. Рассматриваются изгибаемые пластины постоянной толщины $2 h=$ const, нагруженные в поперечном направлении $z$. Предполагается: к рассматриваемому моменту времени деформации ползучести получили настолько значительное развитие, что по сравнению с ними можно пренебречь начальными упругими и пластическими деформациями [1]; прогибы считаются малыми; кирхгофовская

Андрей Петрович Янковский (д.ф.-м.н.), ведущий научный сотрудник, лаб. физики быстропротекающих процессов. 
пластина (допустимость гипотез Кирхгофа при расчёте установившейся ползучести изгибаемых пластин обоснована в [1]) испытывает поперечный изгиб и состоит из изотропной металлической матрицы и внедрённых в неё $N$ семейств тонковолокнистой однородной арматуры (металлических проволок, возможно, изготовленных из разных материалов) постоянного поперечного сечения; пластина по толщине имеет регулярную и квазиоднородную структуру; температура однородна.

Для формулировки задачи РА поперечно изгибаемых пластин, работающих в условиях установившейся ползучести, необходимо использовать общеизвестные уравнения равновесия (см. (1) в [3]) и определяющие соотношения (см. (14), (15) в [3])

$$
\begin{aligned}
& M_{11}=-C_{1111} v,{ }_{11}-C_{1122} v,,_{22}-2 C_{1112} v,,_{12} ; \\
& M_{22}=-C_{1122} v,{ }_{11}-C_{2222} v, 22-2 C_{2212} v,{ }_{12} ; \\
& M_{12}=-C_{1112} v,,_{11}-C_{2212} v, 22-2 C_{1212} v,,_{12},
\end{aligned}
$$

где

$$
\begin{aligned}
& C_{1111}=2 D_{0}\left(1-\sum_{k} \omega_{k}\right) \kappa^{\mu_{0}-1}+\sum_{k} D_{k} \kappa_{k}^{\mu_{k}-1} \omega_{k} \cos ^{4} \psi_{k}, \\
& C_{2222}=2 D_{0}\left(1-\sum_{k} \omega_{k}\right) \kappa^{\mu_{0}-1}+\sum_{k} D_{k} \kappa_{k}^{\mu_{k}-1} \omega_{k} \sin ^{4} \psi_{k}, \\
& C_{1122}=D_{0}\left(1-\sum_{k} \omega_{k}\right) \kappa^{\mu_{0}-1}+\sum_{k} D_{k} \kappa_{k}^{\mu_{k}-1} \omega_{k} \cos ^{2} \psi_{k} \sin ^{2} \psi_{k}, \\
& C_{1112}=\sum_{k} D_{k} \kappa_{k}^{\mu_{k}-1} \omega_{k} \cos ^{3} \psi_{k} \sin \psi_{k}, \\
& C_{2212}=\sum_{k} D_{k} \kappa_{k}^{\mu_{k}-1} \omega_{k} \cos \psi_{k} \sin ^{3} \psi_{k}, \\
& C_{1212}=0,5 D_{0}\left(1-\sum_{k} \omega_{k}\right) \kappa^{\mu_{0}-1}+\sum_{k} D_{k} \kappa_{k}^{\mu_{k}-1} \omega_{k} \cos ^{2} \psi_{k} \sin ^{2} \psi_{k} ; \\
& \\
& \kappa=2 \sqrt{v_{, 11}^{2}+v,{ }_{11} v, 22+v_{, 22}^{2}+v_{, 12}^{2}}, \\
& \kappa_{k}=-v, 11 \cos ^{2} \psi_{k}-v, 22 \sin ^{2} \psi_{k}-v, 12 \sin 2 \psi_{k}, \quad 1 \leqslant k \leqslant N ; \\
& D_{0}=4 \bar{B}_{0} h^{\mu_{0}+2} /\left(\mu_{0}+2\right)=\mathrm{const}, \quad D_{k}=2 \bar{B}_{k} h^{\mu_{k}+2} /\left(\mu_{k}+2\right)=\mathrm{const}, \\
& \bar{B}_{0}=\left(\sqrt{3^{m_{0}+1}} B_{0}\right)^{-\mu_{0}}=\mathrm{const}, \quad \bar{B}_{k}=B_{k}^{-\mu_{k}}=\mathrm{const}, \\
& \mu_{0}=1 / m_{0}, \quad \mu_{k}=1 / m_{k}, \quad 1 \leqslant k \leqslant N ;
\end{aligned}
$$

$B_{0}, m_{0}, B_{k}, m_{k}$ - известные характеристики (константы) материалов связующего и арматуры $k$-того семейства, определяющие степенной закон установившейся ползучести $\xi_{k}=B_{k} \sigma_{k}^{m_{k}}\left(\xi_{k}\right.$ - скорость деформации, $\sigma_{k}$ - напряжение, $0 \leqslant k \leqslant N)$ материалов компонент композиции; $\omega_{k}, \psi_{k}$-плотность и угол (отсчитываемый от направления $x_{1}$ прямоугольной декартовой системы координат $\left.x_{1} x_{2} z\right)$ армирования проволокой $k$-того семейства; $M_{i j}-$ изгибающие и крутящие моменты в пластине; $v$-скорость прогиба установившейся ползучести; нижний индекс после запятой означает частное дифференцирование по соответствующей переменной $x_{i}(i=1,2)$; суммирование производится по указанному индексу от 1 до $N$, если не проставлены пределы.

На верхней $(z=h)$ лицевой поверхности пластины выполняется условие РА для всех семейств волокон (проволок):

$$
\sigma_{k}\left(x_{1}, x_{2}, z\right)=\sigma_{k}^{0}=\mathrm{const} \neq 0, \quad z=h, \quad\left(x_{1}, x_{2}\right) \in G, \quad 1 \leqslant k \leqslant N,
$$


где $\sigma_{k}$ - напряжение в арматуре $k$-того семейства (см. (5) в [3]); $\sigma_{k}^{0}$ - заданное значение. В силу реализации условий поперечного изгиба пластины на нижней лицевой поверхности $(z=-h)$ также будут выполняться условия РА, но только $\sigma_{k}\left(x_{1}, x_{2},-h\right)=-\sigma_{k}^{0}=$ const (см. (3), (5) в [3]).

Так как непрерывные волокна имеют постоянные площади поперечных сечений, параметры армирования $\omega_{k}, \psi_{k}$ связаны уравнением (8) из [3].

Пусть область $G$, занимаемая пластиной в плане, ограничена контуром $\Gamma$, тогда на одной части этого контура (обозначим её $\Gamma_{p}$ ) могут быть заданы общеизвестные статические граничные условия по изгибающему моменту и приведённой поперечной силе Кирхгофа (см. (9), (10) в [3]), а на другой части (обозначим её $\Gamma_{u}$ ) - кинематические условия (см. (11) в [3]):

$$
v\left(\Gamma_{u}\right)=v_{0}, \quad v,_{1} \cos \beta+v,_{2} \sin \beta=\theta_{n}, \quad\left(x_{1}, x_{2}\right) \in \Gamma_{u},
$$

где $v_{0}, \theta_{n}$-скорость прогиба на $\Gamma_{u}$ и производная от скорости прогиба по направлению внешней нормали к контуру, задаваемой углом $\beta$, который откладывается от направления $x_{1}$.

На той части контура $\Gamma$ (обозначим её $\Gamma_{k}$ ), на которой волокна $k$-того семейства входят в область $G$, необходимо задать краевые условия для плотностей армирования [4]:

$$
\omega_{k}\left(\Gamma_{k}\right)=\omega_{0 k}, \quad 1 \leqslant k \leqslant N,
$$

где $\omega_{0 k}$ - заданные на $\Gamma_{k}$ функции.

Разыскиваемая РА-структура должна удовлетворять физическим ограничениям

$$
0 \leqslant \omega_{k} \quad(1 \leqslant k \leqslant N), \quad \sum_{k} \omega_{k} \leqslant \omega_{*} \leqslant 1,
$$

где $\omega_{*}=$ const - предельно допустимая суммарная плотность армирования (на практике $\omega_{*} \approx 0,7$ ).

2. Система разрешающих уравнений и её свойства. Из условия РА (5) и степенного закона установившейся ползучести $\xi_{k}=B_{k} \sigma_{k}^{m_{k}}$ на верхней лицевой поверхности $(z=h)$ получаем постоянство скорости деформаций ползучести в арматуре $k$-того семейства $\left(\xi_{k}\left(x_{1}, x_{2}, h\right)=\xi_{k}^{0}=\right.$ const), поэтому с учётом второго равенства (3) и соотношений (3) из [3] имеем

$$
\xi_{k}\left(x_{1}, x_{2}, h\right)=h \kappa_{k}\left(x_{1}, x_{2}\right)=\xi_{k}^{0}=B_{k}\left(\sigma_{k}^{0}\right)^{m_{k}}=\text { const } \neq 0,
$$

отсюда

$$
\kappa_{k}\left(x_{1}, x_{2}\right)=\kappa_{k}^{0}=\xi_{k}^{0} / h=h^{-1} B_{k}\left(\sigma_{k}^{0}\right)^{m_{k}}=\text { const } \neq 0,
$$

где $h=$ const, $1 \leqslant k \leqslant N$, т.е. скорость параметра искривления пластины $\kappa_{k}$ в направлении армирования проволокой $k$-того семейства в задаче РА, как и в [2], постоянна.

Выражения для моментов (1) с учётом (2), (9) и второго равенства (3) примут вид

$$
\begin{aligned}
& M_{11}\left(x_{1}, x_{2}\right)=-D_{0}\left(1-\sum_{k} \omega_{k}\right) \kappa^{\mu_{0}-1}\left(2 v,{ }_{11}+v, 22\right)+\sum_{k} S_{k} \omega_{k} \cos ^{2} \psi_{k} \\
& M_{22}\left(x_{1}, x_{2}\right)=-D_{0}\left(1-\sum_{k} \omega_{k}\right) \kappa^{\mu_{0}-1}(2 v, 22+v, 11)+\sum_{k} S_{k} \omega_{k} \sin ^{2} \psi_{k} \\
& M_{12}\left(x_{1}, x_{2}\right)=-D_{0}\left(1-\sum_{k} \omega_{k}\right) \kappa^{\mu_{0}-1} v, 12+\sum_{k} S_{k} \omega_{k} \cos \psi_{k} \sin \psi_{k}
\end{aligned}
$$


где

$$
S_{k}=D_{k}\left(\kappa_{k}^{0}\right)^{\mu_{k}}=\text { const } \neq 0, \quad 1 \leqslant k \leqslant N .
$$

Из второго соотношения (3) с учётом (9) получаем условие РА:

$$
v,{ }_{11} \cos ^{2} \psi_{k}+v, 22 \sin ^{2} \psi_{k}+v,{ }_{12} \sin 2 \psi_{k}=-\kappa_{k}^{0}=\text { const } \neq 0 .
$$

Для получения системы разрешающих уравнений подставим выражения для моментов (10) в уравнения равновесия (см. (1) в [3]) и исключим из рассмотрения поперечные силы $F_{1}, F_{2}$, тогда после элементарных преобразований с учётом условия постоянства поперечных сечений волокон (см. (8) в [3]) получим одно уравнение равновесия:

$$
\begin{aligned}
\sum_{k} S_{k}\left[\partial_{n}\left(\psi_{k}, A_{k}\right)-A_{k}^{2} / \omega_{k}\right]- & \\
& -D_{0} \sum_{i=1}^{2}\left[\left(1-\sum_{k} \omega_{k}\right) \kappa^{\mu_{0}-1}\left(2 v,{ }_{i i}+v,{ }_{j j}\right)\right]_{, i i}- \\
- & 2 D_{0}\left[\left(1-\sum_{k} \omega_{k}\right) \kappa^{\mu_{0}-1} v, 12\right]_{, 12}=-p\left(x_{1}, x_{2}\right), \quad j=3-i,
\end{aligned}
$$

где

$$
\begin{gathered}
A_{k}=\omega_{k} \partial_{s}\left(\psi_{k}, \psi_{k}\right), \quad 1 \leqslant k \leqslant N \\
\partial_{s}\left(\psi_{k}, \cdot\right)=(\cdot),,_{1} \cos \psi_{k}+(\cdot),,_{2} \sin \psi_{k}, \\
\partial_{n}\left(\psi_{k}, \cdot\right)=\partial_{s}\left(\psi_{k}+\pi / 2, \cdot\right)=-(\cdot),,_{1} \sin \psi_{k}+(\cdot),{ }_{2} \cos \psi_{k}
\end{gathered}
$$

$A_{k}$ - введенные для удобства функции, имеющие смысл кривизны траекторий РА $k$-того семейства, умноженной на плотность армирования этого семейства; $\partial_{s}\left(\psi_{k}, \cdot\right), \partial_{n}\left(\psi_{k}, \cdot\right)$ - операторы дифференцирования по направлениям $\psi_{k}$ и $\psi_{k}+\pi / 2$ соответственно (т. е. вдоль траекторий армирования волокнами $k$-того семейства и поперек направления армирования); $p$ - распределённая поперечная нагрузка.

K уравнениям (13), (14) следует добавить условия РА (12), которые с учётом (14), (15) примут вид

$$
\partial_{s}\left(\psi_{k}, \partial_{s}\left(\psi_{k}, v\right)\right)-A_{k} \partial_{n}\left(\psi_{k}, v\right) / \omega_{k}=-\kappa_{k}^{0}=\text { const } \neq 0, \quad 1 \leqslant k \leqslant N
$$

где значение $\kappa_{k}^{0}$ определяется формулой $(9)$.

Для окончательного замыкания системы уравнений (13), (14), (16) следует использовать условия постоянства поперечных сечений волокон (см. (8) в [3]), которые с учётом (15) можно записать так:

$$
\partial_{s}\left(\psi_{k}, \omega_{k}\right)+\omega_{k} \partial_{s}\left(\psi_{k}, \psi_{k}\right)=0, \quad 1 \leqslant k \leqslant N .
$$

Для записи статических граничных условий (см. (9), (10) в [3]) в разрешающем виде необходимо выражения для моментов (10) подставить в эти условия и исключить из рассмотрения перерезывающие силы $F_{1}, F_{2}$, тогда после элементарных преобразований с учётом (14), (15), (17) получим 


$$
\begin{aligned}
& \sum_{k} S_{k} \omega_{k} \cos ^{2}\left(\psi_{k}-\beta\right)-D_{0}\left(1-\sum_{k} \omega_{k}\right) \kappa^{\mu_{0}-1}\left[\left(2 v,,_{11}+v,{ }_{22}\right) \cos ^{2} \beta+\right. \\
& \left.+\left(2 v,,_{22}+v,{ }_{11}\right) \sin ^{2} \beta+v,{ }_{12} \sin 2 \beta\right]=M_{n}, \quad\left(x_{1}, x_{2}\right) \in \Gamma_{p} ; \\
& -\sum_{k} S_{k} A_{k} \sin \left(\psi_{k}-\beta\right)+0,5 \sum_{k} S_{k} \partial_{\tau}\left(\omega_{k} \sin 2\left(\psi_{k}-\beta\right)\right)- \\
& -D_{0} \sum_{i=1}^{2} n_{i}\left\{\left[\left(1-\sum_{k} \omega_{k}\right) \kappa^{\mu_{0}-1}\left(2 v,,_{i i}+v,{ }_{j j}\right)\right]_{, i}+\left[\left(1-\sum_{k} \omega_{k}\right) \kappa^{\mu_{0}-1} v,_{12}\right]_{, j}\right\}- \\
& \text { - } D_{0} \sum_{i=1}^{2}(-1)^{i+1} n_{i}\left\{( 1 - \sum _ { k } \omega _ { k } ) \kappa ^ { \mu _ { 0 } - 1 } \left[0,5\left(v, 22-v,{ }_{11}\right) \sin 2 \beta+\right.\right. \\
& \left.\left.+v,{ }_{12} \cos 2 \beta\right]\right\}_{, j}=F_{n z}, \quad j=3-i, \quad\left(x_{1}, x_{2}\right) \in \Gamma_{p},
\end{aligned}
$$

где

$$
n_{1}=\cos \beta, \quad n_{2}=\sin \beta, \quad \partial_{\tau}(\cdot)=\partial_{n}(\beta, \cdot) ;
$$

$M_{n}, F_{n z}$ - изгибающий момент и приведённая поперечная сила Кирхгофа, заданные на $\Gamma_{p} ; \partial_{\tau}(\cdot)$ - оператор дифференцирования вдоль контура пластины $Г$.

Кинематические граничные условия (6) и краевые условия для плотностей армирования (7) остаются без изменения. (На контуре $Г$ могут быть заданы и смешанные из (18), (19), (6) граничные условия, например, условия свободного опирания.)

Таким образом, система разрешающих уравнений рассматриваемой задачи PA состоит из $3 N+1$ уравнений $(13),(14),(16),(17)$ и замкнута относительно неизвестных функций $v, A_{k}, \psi_{k}, \omega_{k}, 1 \leqslant k \leqslant N$. Для однозначного интегрирования системы разрешающих уравнений на кромках пластины должны быть заданы граничные и краевые условия (6), (7), (18), (19).

Полученная система разрешающих уравнений $(13),(14),(16),(17)$ и соответствующие ей статические граничные условия (18), (19) показывают, что задачи определения скорости прогиба установившейся ползучести $v$ и параметров РА $A_{k}, \psi_{k}, \omega_{k}$ связаны и решать их необходимо совместно, причём в целом система уравнений и статические граничные условия существенно нелинейны. Нелинейность имеет двоякое происхождение: во-первых, структурная нелинейность (так как параметры РА $A_{k}, \psi_{k}, \omega_{k}$ - неизвестные функции), во-вторых, физическая нелинейность, так как скорости деформаций ползучести $\xi$ связаны нелинейно (а именно степенным законом) с напряжениями $\sigma$ в каждой фазе композиции. Все это значительно усложняет качественный анализ краевой задачи РА изгибаемых пластин, работающих в условиях установившейся ползучести, и разработку методов её (задачи) решения.

Определим тип системы разрешающих уравнений, используя детерминантный метод [5]. Так как только уравнение (13) содержит старшие производные от неизвестных функций $A_{k}, \omega_{k}, v$, для определения типа системы уравнений необходимо каждое уравнение (16), (17) формально продифференцировать по произвольному направлению (например, по $x_{1}$ ), после чего следует исключить из рассмотрения характеристики, искусственно порождённые 
этим дифференцированием. Характеристическое уравнение преобразованной таким образом системы разрешающих уравнений имеет вид

$$
P\left(x_{2}^{\prime}\right) \prod_{k=1}^{N}\left(\sin \psi_{k}-x_{2}^{\prime} \cos \psi_{k}\right)^{2}=0,
$$

где производная $x_{2}^{\prime}=d x_{2} / d x_{1}$ задаёт направление характеристики; $P\left(x_{2}^{\prime}\right)-$ полином 4-того порядка относительно $x_{2}^{\prime}$, коэффициенты которого зависят от значений неизвестных функций $v,{ }_{i j}$ :

$$
\begin{aligned}
& P\left(x_{2}^{\prime}\right)=\kappa^{2}\left(1+x_{2}^{\prime 2}\right)^{2}+\left(\mu_{0}-1\right)\left(\left(2 v,,_{11}+v,,_{22}\right)^{2} x_{2}^{\prime 4}+\right. \\
& +2\left[\left(2 v,,_{11}+v,,_{22}\right)\left(2 v, 22+v,{ }_{11}\right)+2 v_{, 12}^{2}\right] x_{2}^{\prime 2}-4 v,,_{12}\left(2 v,,_{11}+v,,_{22}\right) x_{2}^{\prime 3}- \\
& \left.\quad-4 v,,_{12}\left(2 v,,_{22}+v,{ }_{11}\right) x_{2}^{\prime}+\left(2 v,,_{22}+v,,_{11}\right)^{2}\right) .
\end{aligned}
$$

Сомножители, стоящие в (21) под знаком произведения, указывают на то, что система разрешающих уравнений имеет $N$ действительных двукратных характеристик, совпадающих с траекториями РА. Так как для реальных металлов $\mu_{0}-1<0$ (см. (4)), полином $P\left(x_{2}^{\prime}\right)$ в зависимости от значений производных $v,{ }_{i j}$ (т. е. в зависимости от коэффициентов) может иметь разное количество действительных корней в разных точках области $G$. Следовательно, система разрешающих уравнений задачи РА изгибаемых пластин является квазилинейной системой смешанно-составного типа [6].

3. Некоторые свойства решения задачи РА изгибаемых металлокомпозитных пластин. В настоящее время теория систем уравнений смешанно-составного типа разработана весьма неполно [6], чтобы можно было аналитически исследовать в общем виде свойства решения системы разрешающих уравнений задачи PA (13), (14), (16), (17). Однако некоторые весьма важные особенности решения этой системы всё же удалось установить.

Повторяя дословно рассуждения, приведённые в [7] и касающиеся условия постоянства поперечных сечений волокон (17), можно получить следующие результаты:

1) если в краевых условиях (7) задаваемая функция $\omega_{0 k}>0$, то всюду в пластине $\omega_{k}>0$ (т. е. в этом случае при решении задачи РА необходимо контролировать выполнение лишь последнего физического ограничения (8));

2) семейство траекторий армирования волокнами постоянного поперечного сечения не может иметь огибающей и не может асимптотически приближаться к некоторой кривой, в частности, такие волокна (проволоки) не могут по касательным направлениям приближаться к кромкам пластины и не могут пересекаться.

Покажем, что для некоторых типов закрепления пластины невозможно существование гладкого решения исследуемой задачи РА. Для этого рассмотрим условие РА в исходной форме (12) и два неравенства:

$$
\begin{gathered}
v,{ }_{11} \cos ^{2} \delta+v,{ }_{22} \sin ^{2} \delta+v,,_{12} \sin 2 \delta \geqslant 0 \\
v,{ }_{11} \cos ^{2} \delta+v,{ }_{22} \sin ^{2} \delta+v,,_{12} \sin 2 \delta \leqslant 0, \quad \delta \in[0,2 \pi) .
\end{gathered}
$$


Если в некоторой точке $\left(x_{1}, x_{2}\right) \in G$ выполняется условие $(23)$ при произвольном угле $\delta$, то в её окрестности поверхность $V$, определяемая функцией $v\left(x_{1}, x_{2}\right)$, лежит выше касательной плоскости, проведённой через точку $\left(x_{1}, x_{2}, v\right)[8]$. Аналогично, если в некоторой точке выполняется условие (24) при произвольном угле $\delta$, то в её окрестности поверхность $V$ располагается ниже касательной плоскости.

Предположим, что разыскиваются гладкие решения задачи РА, тогда условие (12) при фиксированном $\kappa_{k}^{0} \neq 0$ должно выполняться всюду в пластине. Следовательно, в этом случае не могут выполняться одновременно условия (23), (24) в разных подобластях пластины, что вытекает из (12) при задании $\delta=\psi_{k}$. Поэтому, если разыскиваются гладкие решения задачи РА, функция $v\left(x_{1}, x_{2}\right)$ образует такую поверхность $V$, которая не может в окрестности одних точек $\left(x_{1}, x_{2}, v\right)$ лежать выше касательной плоскости, а в окрестностях других точек - ниже. В частности, $v\left(x_{1}, x_{2}\right)$ не может достигать одновременно локальных максимумов и минимумов. Действительно, в точках локальных максимумов должны выполняться условия (24), а в точках локальных минимумов - условия (23).

Проведённые рассуждения позволяют заключить, что гладкие решения задачи РА поперечно изгибаемых пластин не могут существовать при следующих типах закрепления:

1) по всей кромке Г пластина жёстко защемлена;

2) пластина оперта по всей кромке $Г$ и хотя бы в одной точке защемлена;

$3)$ на одной части кромки $\left(\Gamma_{0}\right)$ пластина оперта, на другой $\left(\Gamma_{z}\right)$ жёстко защемлена, а на третьей $\left(\Gamma_{f}\right)$ задана податливая опора с защемлением (при этом часть кромки $\Gamma_{0}$ может вообе отсутствовать).

При двух первых типах закрепления пластины на всей кромке $\Gamma$ выполняется первое кинематическое условие $(6)$ при $v_{0}=0$, а значит, при $v \neq 0$ внутри области $G$, занимаемой пластиной в плане, обязательно найдётся точка $A$, в которой будут реализовываться условия локального максимума или минимума. Пусть для простоты в точке $A$ реализуется условие локального максимума и других локальных экстремумов внутри $G$ нет, тогда на части кромки $\Gamma_{z}$, которая жёстко защемлена, найдётся точка $B$, в которой будет реализовываться условие локального минимума. Но одновременное наличие точек локального максимума и минимума невозможно при гладких решениях задачи РА, что приводит к противоречию. Для третьего типа закрепления точка $A$ может лежать не внутри области $G$, а на кромке $\Gamma_{f}$, точка же $B-$ по-прежнему на кромке $\Gamma_{z}$, что вновь приводит к противоречию.

Очевидно, что при указанных типах закрепления пластины в окрестностях точек локальных минимумов функции $v\left(x_{1}, x_{2}\right)$ необходимо выполнение неравенств $\kappa_{k}^{0}<0$, а в окрестностях точек её локальных максимумов $\kappa_{k}^{0}>0$ $\left(\kappa_{k}^{0}=\right.$ const $\left.\neq 0\right)$, что следует из сравнения (12) с (23), (24) соответственно. Значит, в этих случаях нужно использовать сопряжённые (разрывные) решения задачи РА. При этом найдутся такие линии контакта $\Gamma_{u}^{v}$ подобластей $G_{u}$, $G_{v}(\subset G)$, на которых напряжения в равнонапряжённых волокнах на верхней (нижней) лицевой поверхности пластины будут иметь разные знаки по разные стороны от $\Gamma_{u}^{v}$. Но тогда из условия стыковки по изгибающему моменту на линии $\Gamma_{u}^{v}$ (это условие представляет собой равенство, в правой и левой частях которого стоят выражения, аналогичные тому, что приведено в левой 
части граничного условия (18)) следует, что в силу малости параметра $D_{0} / D_{1}$ (см. (4), (11) и левую часть равенства (18)) на этой линии $\Gamma_{u}^{v}$ скорости деформаций установившейся ползучести могут достигать по модулю больших значений, т. е. в окрестности указанных линий стыковки $\Gamma_{u}^{v}$ в связующем может возникнуть локальный эффект типа краевого.

Возможность появления краевого эффекта в связующем в задачах РА изгибаемых металлокомпозитных пластин была показана ранее на конкретном

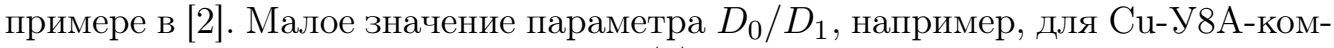
позиции непосредственно следует из (4) и конкретных значений величин $B_{0}$, $m_{0}, B_{1}, m_{1}$ для этой композиции (см. (35) в [2]). Следовательно, рассматриваемая задача РА является задачей с сингулярным возмущением, и решение её может обладать локальными и краевыми эффектами в связующем (арматура всюду равнонапряжена, поэтому в ней такие эффекты не возникают).

Проанализируем теперь зависимость частных производных функции $v$ от угла, под которым равнонапряжённые волокна пересекают жёстко защемлённую кромку $\Gamma_{z}$. Пусть кривая $\Gamma_{z}$ имеет требуемую степень гладкости, тогда в некоторой её окрестности можно ввести криволинейную ортогональную систему координат такую, что линия $\Gamma_{z}$ будет принадлежать одному из семейств координатных линий. В этом случае на $\Gamma_{z}$ дифференцирование вдоль линии семейства, которому принадлежит $\Gamma_{z}$, будет осуществляться оператором $\partial_{\tau}(\cdot)($ см. $(20))$, а вдоль линий ортогонального семейства - оператором $\partial_{s}(\beta, \cdot)($ см. (15)), где $\beta$ - угол, задающий направление внешней нормали к $\Gamma_{z}$. Тогда частные производные от произвольной функции $f$ по переменным $x_{1}, x_{2}$ на $\Gamma_{z}$ имеют выражение

$$
f,{ }_{1}=\cos \beta \partial_{s}(\beta, f)-\sin \beta \partial_{\tau}(f), f,_{2}=\sin \beta \partial_{s}(\beta, f)-\cos \beta \partial_{\tau}(f) .
$$

Так как на $\Gamma_{z}$ задано жёсткое защемление, то имеют место кинематические граничные условия (см. (6), (15))

$$
v\left(\Gamma_{z}\right)=0, \quad \partial_{s}\left(\beta, v\left(\Gamma_{z}\right)\right)=0,
$$

откуда

$$
\partial_{\tau}^{m}\left(v\left(\Gamma_{z}\right)\right)=0, \quad \partial_{\tau}^{l}\left(\partial_{s}\left(\beta, v\left(\Gamma_{z}\right)\right)\right)=0,
$$

где $m, l$ определяют порядок повторного дифференцирования.

Рассмотрим условие PA (12) на линии $\Gamma_{z}$, заменив все частные производные по формулам (25), тогда после элементарных преобразований с учётом равенств (26), (27) получим

$$
\partial_{s}^{2}\left(\beta, v\left(\Gamma_{z}\right)\right)=-\kappa_{k}^{0} / \cos ^{2}\left(\psi_{k}-\beta\right), \quad \kappa_{k}^{0}=\mathrm{const} \neq 0,
$$

Отсюда следует, что при $\cos \left(\psi_{k}-\beta\right) \rightarrow 0\left|\partial_{s}^{2}\left(\beta, v\left(\Gamma_{z}\right)\right)\right|$ неограниченно возрастает (со вторым порядком роста [8]), а значит, чем меньше угол между направлением траектории PA и касательной к кромке $\Gamma_{z}$, тем больше по модулю вторые производные от скорости прогиба установившейся ползучести и, как следствие, напряжения в связующем, которые возрастают по модулю неограниченно при стремлении этого угла к нулю (см. (3), (5) в [3]).

Если продифференцировать условие РА $(12)$, применив оператор $\partial_{s}(\beta, \cdot)$, то после преобразований с учётом (28) получим, что при $\psi_{k}-\beta \rightarrow \pm \pi / 2$ на 
кромке $\Gamma_{z}$ третьи и четвёртые производные от скорости прогиба $v$ также неограниченно возрастают по модулю, имея при этом четвёртый и шестой порядок роста соответственно.

Эти особенности решения рассматриваемой задачи РА необходимо учитывать при её численном интегрировании, так как конечно-разностные аналоги соответствующих уравнений в силу указанных выше причин могут в окрестности кромки $\Gamma_{z}$ вносить большие ошибки аппроксимации, что может существенно отразиться на всём численном решении.

Исследуем поведение траекторий РА в окрестности некоторых точек пластины, закрепленной одним из указанных выше способов. Для этого рассмотрим условия РА волокон $k$-того семейства (16) в криволинейной системе координат: одно семейство координатных линий пусть совпадает с траекториями армирования $k$-того семейства (оператор дифференцирования вдоль них $\left.\partial_{s k}(\cdot) \equiv \partial_{s}\left(\psi_{k}, \cdot\right)\right)$, а другое семейство - с линиями, ортогональными к траекториям РА (оператор дифференцирования вдоль них $-\partial_{n k}(\cdot) \equiv \partial_{n}\left(\psi_{k}, \cdot\right)$, см. (15)). Для удобства дальнейшего изложения условие РА (16) перепишем в виде

$$
\partial_{s k}^{2}(v)-\rho_{k}^{-1} \partial_{n k}(v)=-\kappa_{k}^{0}=\text { const } \neq 0, \quad 1 \leqslant k \leqslant N,
$$

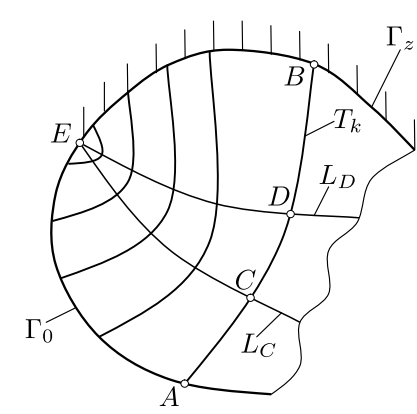

$\mathrm{K}$ вопросу об особенностях поведения траекторий равнонапряжённого армирования в окрестности точки сопряжения разных типов опирания изгибаемой пластины где $\rho_{k}^{-1}=\partial_{s k}\left(\psi_{k}\right) \equiv \partial_{s}\left(\psi_{k}, \psi_{k}\right)$ - кривизна траектории РА.

Пусть на кромке $Г$, ограничивающей пластину, существует точка $E$, которая «разбивает» $\Gamma$ на две части $\Gamma_{0}$ и $\Gamma_{z}$, причём на $\Gamma_{0}$ пластина оперта (т.е. задано первое граничное условие (6) при $\left.v_{0}=0\right)$, а на $\Gamma_{z}$ пластина жёстко защемлена (т. е. заданы оба граничных условия (6) при $\left.v_{0}=0, \theta_{n}=0\right)$. Предположим, что волокна входят в пластину на части кромки $\Gamma_{0}$, а выходят на $\Gamma_{z}$ (см. рисунок). Выберем конкретную траекторию армирования $T_{k}$, которая входит в пластину в точке $A \in \Gamma_{0}$, а выходит в точке $B \in \Gamma_{z}$. Тогда из условия непересечения волокон одного и того же семейства получим, что все волокна, входящие в пластину на криволинейном отрезке $A E \subset \Gamma_{0}$, будут обязательно выходить из неё на отрезке $B E \subset \Gamma_{z}$.

Исследуем поведение скорости прогиба установившейся ползучести $v$ вдоль траектории армирования. Так как $v\left(\Gamma_{0}\right)=0, v\left(\Gamma_{z}\right)=0$, по теореме Ролля [8] для гладкой вдоль траектории $T_{k}$ функции $v$ в некоторой точке $C \in T_{k}$ обязательно выполняется условие

$$
\partial_{s k}(v)=0 .
$$

Но в точке $B \in \Gamma_{z}$ в силу жёсткого защемления части кромки $\Gamma_{z}$ выполняется такое же условие (30). Поэтому, применяя ещё раз теорему Ролля, на отрезке $C B \subset T_{k}$ найдём такую точку $D$, в которой

$$
\partial_{s k}^{2}(v)=0 .
$$


Очевидно, что для всех волокон, входящих в пластину на отрезке $A E$ и выходящих на $B E$, можно построить такие точки $C, D$, которые образуют линии $L_{C}, L_{D}$, стремящиеся к точке $E$, причём вдоль кривой $L_{C}$ выполняется равенство (30), а вдоль линии $L_{D}$ - равенство (31). С другой стороны, в окрестности точки $E$ в силу жёсткого защемления части кромки $\Gamma_{z}$ будут иметь место приближённые равенства

$$
v,_{1} \approx v,_{2} \approx 0 \Rightarrow \partial_{s k}(v) \approx \partial_{n k}(v) \approx 0,
$$

поэтому при стремлении к точке $E$ вдоль кривой $L_{D}$ получим в условиях $\mathrm{PA}$ (29) следующие значения для производных

$$
\partial_{s k}^{2}(v)=0, \quad \partial_{n k}(v) \rightarrow 0 .
$$

Выполнение же условий (29) с учётом (32) и $\kappa_{k}^{0} \neq 0$ возможно лишь при $\left|\rho_{k}^{-1}\right| \rightarrow \infty$ в точке $E$. Следовательно, при рассматриваемом типе закрепления пластины и указанном способе укладки арматуры в окрестности точки $E$ кривизна траекторий армирования должна быть неограниченной по модулю. Если продифференцировать условие РА в форме (12), применив оператор $\partial_{s k}(\cdot)$, то при условии $\left|\rho_{k}^{-1}\right| \rightarrow \infty$ получим неограниченное по модулю возрастание третьих производных от скорости прогиба $v$, что может вызвать большие по модулю значения вторых производных от $v$, а значит, и большие значения напряжений в связующем в окрестности точки $E$ (см. (3), (5) в [3]).

В заключение настоящего исследования остановимся на вопросе о неединственности решения задачи РА изгибаемых пластин. Действительно, статические (18), (19) и кинематические (6) граничные условия являются естественными условиями в задачах поперечного изгиба пластин и определяются конкретными условиями эксплуатации конструкции. Краевые же условия для плотностей армирования (7) являются «технологическими» условиями, фактически определяющими, какое количество волокон $k$-того семейства вводится в пластину на данном участке кромки. Выбор количества вводимых в пластину волокон является в определённой степени произвольным, зависящим от воли проектировщика и удовлетворяющим лишь физическим ограничениям (8) и условиям существования соответствующего проекта РА. Следовательно, задача РА обладает функциональными произволами $\omega_{0 k}\left(\Gamma_{k}\right)$, связанными с краевыми условиями (7), и чем больше количество семейств арматуры, внедряемой в пластину, тем больше количество этих произволов. Варьируя функции $\omega_{0 k}\left(\Gamma_{k}\right)$ в краевых условиях $(7)$, можно получать целые «пучки» решений задачи РА, из которых можно выбирать проекты с определёнными свойствами, например, с наименьшим расходом армирующих волокон, или с наименьшей податливостью в условиях установившейся ползучести, или наиболее удобные с точки зрения технологической реализации. Это означает, что РА-проектами можно целенаправленно управлять. (В [2] целесообразность такого управления продемонстрирована на конкретном примере осесимметрично нагруженной кольцевой пластины.)

Кроме того, в силу существенной нелинейности статических граничных условий $(18),(19)$ и условий РА (12) относительно функций $\psi_{k}, \omega_{k}, A_{k}$ задача PA может иметь несколько альтернативных решений даже при фиксированных функциях $\omega_{0 k}\left(\Gamma_{k}\right)$ в краевых условиях (7). Так, например, условия 
PA (12) определяют два направления армирования $\psi_{k}$, симметричных относительно главных направлений скоростей деформаций ползучести (в этом легко убедиться, положив в $(12) v, 12=0$, что соответствует главным направлениям); в [2] на конкретном примере была показана возможность существования двух альтернативных решений задачи РА при одних и тех же краевых условиях (7).

Все это дополнительно расширяет «спектр» решений задачи РА, из которого можно выбрать проекты, наиболее значимые для практического использования.

Работа выполнена при поддержке РФФИ (проект № 10-01-90402-Укр) и Президиума СО РАН (постановление № 10 от 15.01.09, проект № 10).

\section{БИБЛИОГРАФИЧЕСКИЙ СПИСОК}

1. Качанов Л. М. Теория ползучести. М.: Физматгиз, 1960. 456 с.; англ. пер.: Kachanov L. M. Theory of creep. Boston, MA: Nat. Lending Lib., 1958. 430 pp.

2. Янковский А.П. Равнонапряжённое армирование кольцевых изгибаемых металлокомпозитных пластин, работающих в условиях установившейся ползучести // Becmн. Сам. гос. техн. ун-та. Сер. Физ.-мат. науки, 2010. № 5(21). С. 42-54. [Yankovskii A. P. Equalstress reinforcement the ring bending metal-composites plates working in conditions of steady creep // Vestn. Samar. Gos. Tekhn. Univ. Ser. Fiz.-Mat. Nauki, 2010. no. 5(21). Pp. 42-54].

3. Немировский Ю. В., Янковский А. П. Установившаяся ползучесть слоисто-волокнистых изгибаемых металлокомпозитных пластин // Вестн. Сам. гос. техн. ун-та. Сер. Физ.мат. науки, 2008. № 2(17). C. 66-76. [Nemirovskii Yu. V., Yankovskii A. P. The steady creep layer-fibrous bendings metal-composites plates // Vestn. Samar. Gos. Tekhn. Univ. Ser. Fiz.Mat. Nauki, 2008. no. 2(17). Pp. 66-76].

4. Немировский Ю.В., Янковский А.П. О некоторых особенностях уравнений оболочек, армированных волокнами постоянного поперечного сечения // Механика композиционных материалов и конструкций, 1997. Т. 3, № 2. С. 15-39. [Nemirovskii Yu. V., Yankovskii A. P. On some features of the equations of shells reinforced with fibres of constant crosssection // Mekhanika Kompozitsionnykh Materialov i Konstrukciy, 1997. Vol.3, no. 2. Pp. 15-39].

5. Петровский И. Г. Лекции об уравнениях с частными производными. М.: Физматгиз, 1961. 400 c. [Petrovskiy I. G. Lectures on partial differential equations. Moscow: Fizmatgiz, 1961. 400 pp.]

6. Джураев Т. Д. Краевые задачи для уравнений смешанного и смешанно-составного типов. Ташкент: Фан, 1979. 239 с. [Dzhuraev T. D. Boundary value problems for equations of mixed and mixed-composite types. Tashkent: Fan, 1979. 239 pp.]

7. Немировский Ю. В., Янковский А. П. О некоторых свойствах решений плоских термоупругих задач рационального армирования композитных конструкций// ПММ, 1997. T. 61, № 2. C. 312-321; англ. пер.: Nemirovskii Yu. V., Yankovskii A.P. On some properties of solutions of plane thermoelastic problems of rational reinforcement of composite constructions. // J. Appl. Math. Mech., 1997. Vol.61, no. 2. Pp. 301-309.

8. Фихтенгольи, Г. М. Курс дифференциального и интегрального исчисления. Т. 1. Л.: Физматгиз, 1958. 608 с. [Fikhtengol'ts G. M. A course of differential and integral calculus. Vol. 1. Leningrad: Fizmatgiz, 1958. 608 pp.]

Поступила в редакцию $31 / \mathrm{I} / 2011$;

в окончательном варианте - 18/V/2011. 
MSC: 74K20, 74R20

\section{ON SOME PROPERTIES OF EQUAL-STRESS PROBLEM SOLUTION REINFORCEMENT BENDING THE METAL-COMPOSITE PLATES WORKING IN STEADY CREEP CONDITIONS}

\section{A. P. Yankovskii}

Khristianovich Institute of Theoretical and Applied Mechanics, Siberian Branch of the Russian Academy of Science,

4/1, Institutskaya str., Novosibirsk, 630090.

E-mail: nemirov@itam.nsc.ru

Solution of a stress condition of stochastic heterogeneous plate problem was obtained on the basis of statistic linearization of determinative creep equation and by using a method of spectral representation of random functions. Stochasticity is introduced into determinative creep equation by random function of two variables. It was proved, that stochastic nonhomogeneities of material can lead to significant fluctuations of stress fields.

Key words: metal-composites, equal-stress reinforcement, bending plates, steady creep, properties of solution, qualitative analysis.

Original article submitted 31/I/2011; revision submitted $18 / \mathrm{V} / 2011$.

Andrey P. Yankovskii (Dr. Sci. (Phys. \& Math.)), Leading Research Scientist, Lab. of Fast Processes Physic. 